\title{
Development of an integrated approach to the selection of remediation measures and environmental technologies for their implementation
}

\author{
Vadim Bespalov ${ }^{1}$ and Oksana Gurova ${ }^{1, *}$ \\ ${ }^{1}$ Don State Technical University, 1, Gagarina sq., 344022, Rostov-on-Don, Russia
}

\begin{abstract}
The article is devoted to the scientific substantiation of the formation of a comprehensive methodology for the selection of highly effective and economical remediation measures. To do this, the authors have gradually carried out studies of factors affecting the environment, assessing the levels of their environmental hazard, analyzing the goals and main tasks of the international environmental monitoring system with a proposal for the basic principles of its improvement, and also proposed a new classification of environmental measures aimed at suppressing environmental impacts factors.
\end{abstract}

\section{Introduction}

At the preliminary stage of research, we gave a characteristic of factors negatively affecting the environment. The analysis of long-term observations made it possible to conclude $[1,2,3]$ that in the current conditions of the development of the world economy, the properties of these factors are subject to continuous changes, leading to their negative environmental activity.

At the same time, all factors polluting the environment are divided $[3,4]$ into three main groups:

- the 1st group includes pollutants (material pollution), which can be in solid, liquid and gaseous state;

- the 2nd group includes physical fields, the properties of which depend on the physical nature (acoustic (sound) field, vibration field, thermal field, electromagnetic field, radiation field);

- the 3rd group includes biological pollution, which can be represented by various microorganisms (viruses and bacteria) that worsen the state of ecosystems and negatively affect humans, animals and plants.

It should be noted that the listed factors not only negatively affect the human body, but also worsen the ecological situation both at the global and at the territorial levels, leading also to the premature decommissioning (wear) of the complex of urban facilities and causing serious damage to the utility and economic potential of major cities, urban

\footnotetext{
*Corresponding author: okgurova@yandex.ru
} 
agglomerations and megalopolises with the appropriate infrastructure $[1,2,4,5]$. To identify the degree of environmental degradation, it is necessary to assess the level of impact of the factors described above.

\section{Materials and Methods}

The assessment of the level of environmental hazard of factors affecting the environment is carried out on the basis of a methodology that includes four main stages [6,7]:

1. Allocation for consideration and further analysis of the relevant factor that has a negative impact on the environment.

2. Measurement of the actual level of impact of the factor under consideration.

3. Identification (search) of the normative value of the impact level of the factor under consideration with the help of the relevant normative documents.

4. Comparison of the actual impact level of the factor under consideration with the normative value and assessment of the impact level from the conditions of exceeding or not exceeding the norm.

Based on the results of the assessment of the level of impact of the factor under consideration, and, ultimately, of the group of factors, it is possible to assess the degree of environmental safety (or danger) of the area under consideration:

- if the actual level of impact of a certain factor does not exceed the standard value, it is considered that this factor (pollutant, or physical field, or biological impact) does not have a negative impact on the environment;

- if the actual level of impact of a certain factor exceeds the standard value, it is considered that this factor has a negative impact on the environment to a certain extent. It is in such cases that it is necessary to use systems and complexes for ensuring environmental safety - environmental technologies.

Such an assessment should be carried out continuously and in fact across the entire spectrum of negatively affecting the environment, which is currently being carried out on the basis of the global environmental monitoring system [6,7]. At the international level, such a system operates under the auspices of the World Environmental Monitoring Center UNEP-WCMC, which provides for:

- coordination of the development and implementation of programs for monitoring the state of the environment;

- regulation and control of collection and processing of reliable and comparable data;

- storage and analysis of information, maintenance of special data banks, their coordination, creation of an interstate environmental information system;

- activities to assess and predict the state of environmental objects, natural resources, responses of ecological systems and human health to anthropogenic impact;

- availability of integrated environmental information to a wide range of consumers.

The main tasks of the international environmental monitoring system are $[7,8]$ :

- carrying out, with a certain spatial and temporal resolution, observations of changes in the state of the environment and ecosystems, as well as sources of anthropogenic impacts;

- carrying out assessments of the state of the environment, ecosystems of the country's territory, sources of anthropogenic impact;

- forecasting the state of the environment, ecological situation on the territory of the respective country and its regions, levels of anthropogenic impact under different conditions of the location of productive forces, social and economic scenarios for the development of this country.

However, at present, research continues in the field of development and improvement of the monitoring system with the participation of various international environmental organizations based on the implementation of the following most important principles for 
organizing environmental monitoring [7, 8]: complexity, systematicity, uniformity, continuity, problematic organization (when the research and observation program unfolds only for a specific problem), development (providing for a system of feasibility, that is, implementation and completion of projects, as well as creation of new ones), management priority (when one of the monitoring results is knowledge transferred for decision-making), integrity (ensuring the continuity of the triad "management - monitoring - forecast "), information transparency (when a necessary condition for efficiency, all the results of environmental research and observations should be available to managers, entrepreneurs, politicians, the general public), efficiency (providing for the prompt processing and issuance of information, as well as decision-making).

One of the most important scientific problems is the problem of adaptability of the monitoring system, and one of the difficulties in this case is the need for objective metrological support. Predicting environmental pollution, obtaining reliable information about its state is possible only if there is a sufficiently large array of data, which sets the task of continuous monitoring of environmental pollution levels using automatic measuring instruments. In this case, the reliability of information about the state of the environment must be ensured in two cases: when its initial state is being investigated, and when it is of interest to change its state in comparison with the initial one.

\section{Results and Discussion}

The problems mentioned above, as well as our analysis of the construction of national monitoring systems, allowed us to propose the main directions for improving the international system for monitoring and assessing the level of environmental hazard of factors affecting the environment. In our opinion, these areas should correspond to the implementation of the following five main stages:

Stage 1: the main tasks of the system for monitoring and assessing the level of environmental hazard of factors affecting the environment are accounting, accumulation and analysis of the information received for further informed choice of measures to ensure the environmental safety of territories and forecasting the environmental situation. At the same time, one of the main requirements is to ensure the possibility of receiving and processing information in real time. Moreover, in the process of monitoring, information should be accumulated, allowing to identify and assess the main factors of pollution.

Stage 2: taking into account the tasks and the existing experience in the functioning of the monitoring system, the structure of the main subdivisions of the monitoring network is quickly determined, including the types of observation posts (mobile and stationary) based on the choice of their optimal ratio:

- priority use of inter-territorial stationary posts operating for a long time according to a relatively unchanged program;

- priority use of regional posts operating under relatively short-term survey programs to identify the spatial characteristics of environmental pollution;

- priority use of intensive local posts for observations in very limited areas, which are of the greatest interest from the standpoint of environmental pollution.

Stage 3: It involves solving problems on the feasibility and scale of using automated, remote and other monitoring subsystems, as well as the optimal ratio of stationary and mobile observation posts, for which we have developed methods and general principles for conducting observations, which are presented in the form of guidelines for the main activities.

Stage 4: involves the development of a system for obtaining information, implementing control of its reliability and providing it to consumers on the basis of determining the features of the hierarchical structure of receiving and collecting information "observation 
points - regional information centers - national information center - international information center".

Stage 5: involves the creation of a system for checking the received information for compliance with the initial requirements and, if necessary, further improvement of the monitoring system:

- if the information received meets the requirements for it, the monitoring system can be left unchanged;

- if these requirements are not met in full, as well as when new tasks appear, the monitoring system must continue to be improved while ensuring high operational efficiency, maximum coverage of the surveyed and observed territory, as well as the commensurability of the information received with existing state and international regulations.

We also consider it expedient to introduce the principle of adaptive structure as one of the fundamental principles of organizing the monitoring system (Figure 1), the essence of which is that, based on the analysis of the data received by the monitoring system, it can change its organization: the location of the links of the information-measuring network, rules of their work and information processing procedures.

Consistent implementation of the identification of factors affecting the environment, assessment of the level of their impact on the basis of the monitoring system, zoning of territories according to the level of environmental hazard allows, firstly, to identify ecologically problematic zones (areas) in the territories under consideration and, secondly, specifically for such zones propose appropriate sets of measures to ensure environmental safety, that is, those of an environmental nature.

In modern world practice of the development and implementation of such activities, a rather multifaceted, diverse, sometimes contradictory terminology has developed (techniques, measures, activities, technologies, systems, stages, processes, functional elements, structural elements, etc.), leading to the fact that that specialists of different profiles (ecologists, chemists, biologists, technologists, engineers of various fields and others) understand approaches to ensuring environmental safety and protecting the environment in different ways. This situation is greatly aggravated by the lack of clear, explainable and scientifically grounded classifications on various grounds of the entire complex of environmental measures. 


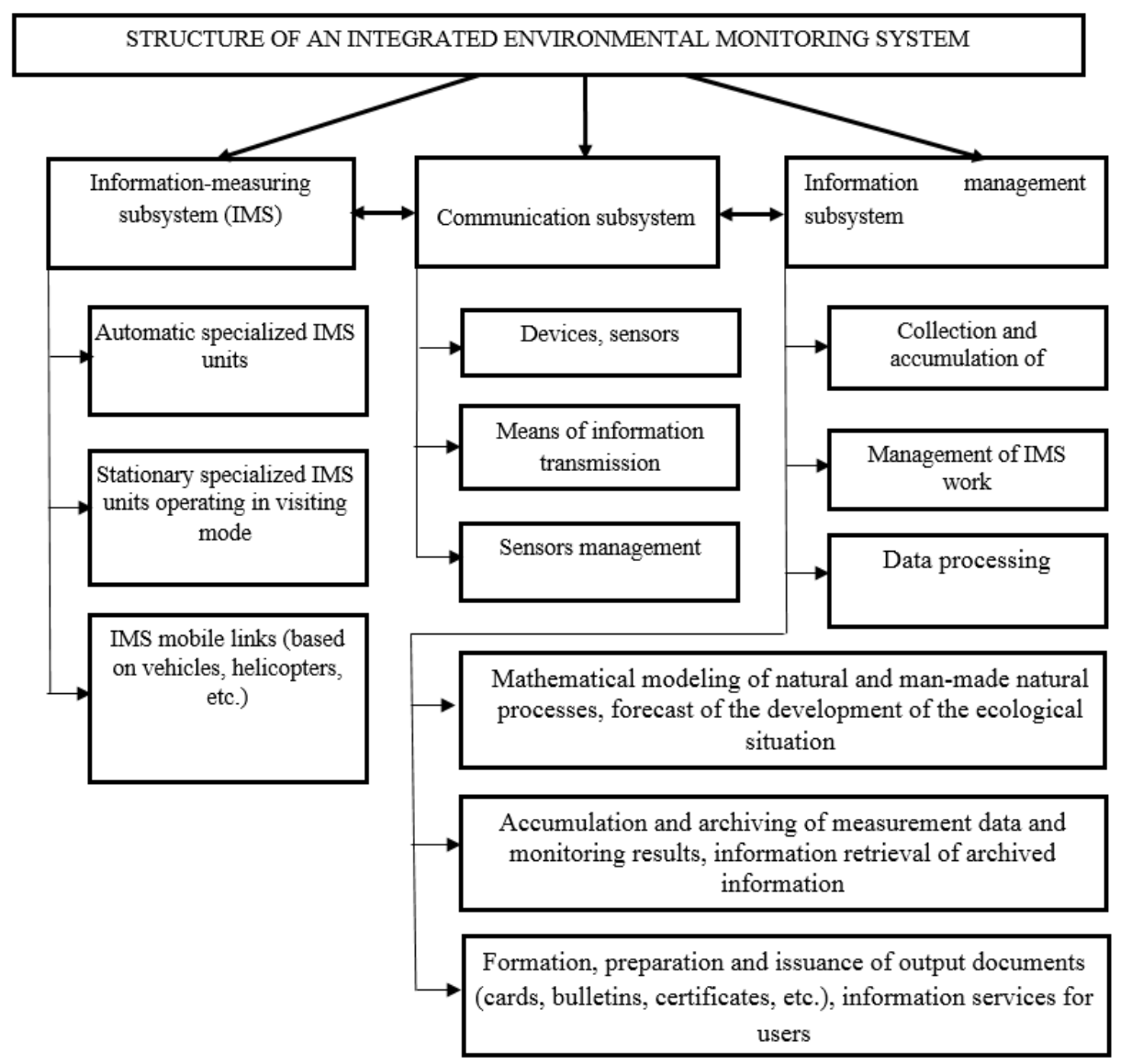

Fig. 1. Structure of the improved integrated system of environmental monitoring and assessment of the level of environmental hazard of factors affecting the environment.

That is why, first of all, we have proposed environmental measures aimed at suppressing factors affecting the environment, divided into three main groups (Figure 2):

- organizational and architectural and planning measures, implying at the level of planning schemes, plans for the future development of territories and the construction of new facilities, the spatial and temporal dispersal of sources of negative factors and impacts affecting the environment (for example, the organization of "green streets" for road traffic; construction of transport junctions, overpasses, crossings, etc; aerodynamically ventilated layouts of city blocks, courtyard spaces and other elements of urban development); 


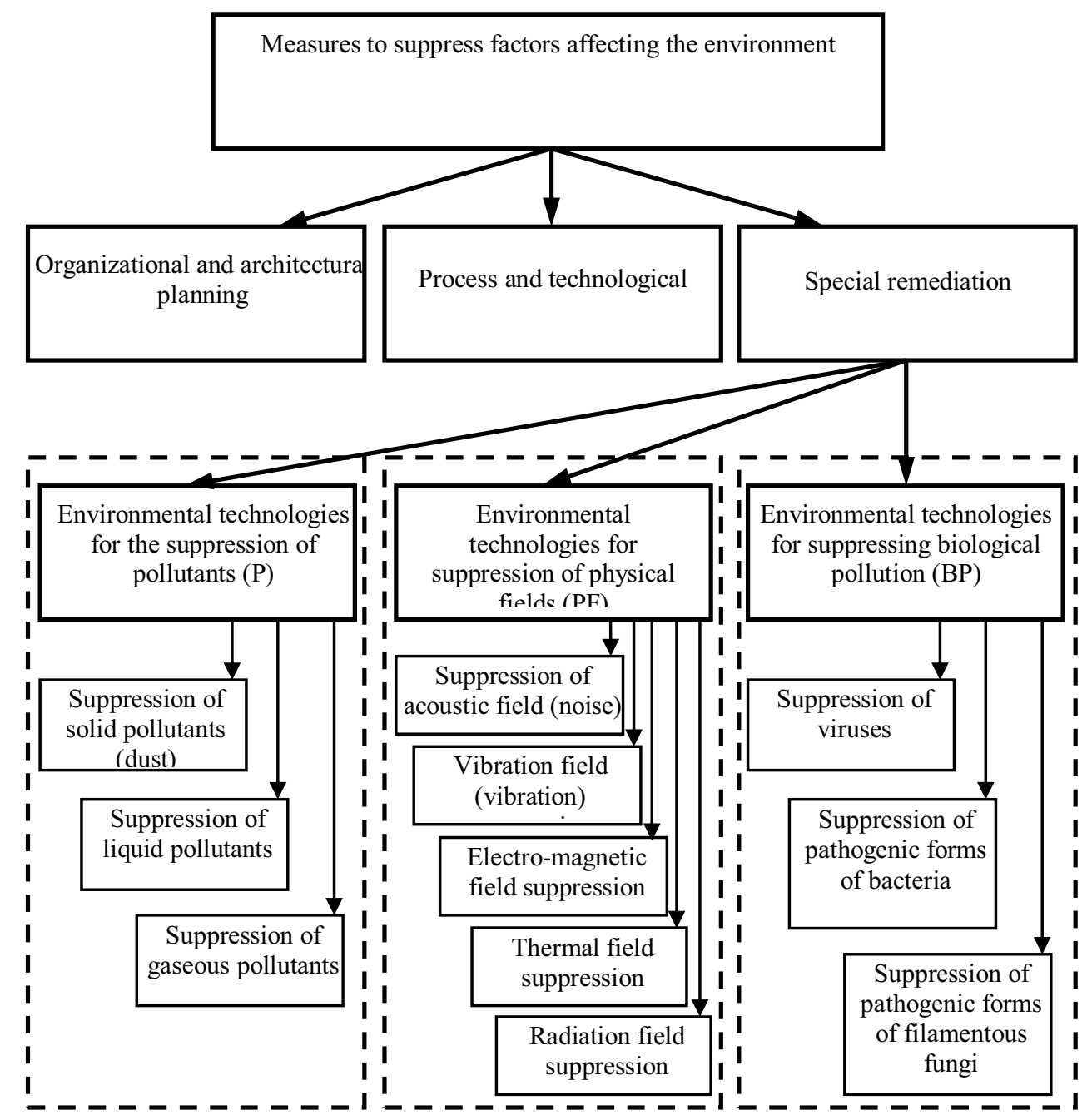

Fig. 2. A set of measures aimed at suppressing factors affecting the environment.

- process and technological measures, implying such optimization of the sequence of operations and actions, leading to the receipt of any completed product, semi-finished product, scheme, innovation system, digital technologies and others, which would ensure minimization of the environmental impact of the corresponding negative factors to the level of normative values (for example, changing the technology of a vehicle by transferring it to another type of fuel "from gasoline to gas", by replacing an internal combustion engine with an electric motor, etc; changing the firing technology of brick production to a technology of plastic molding and drying, etc.);

- special remediation measures, involving the provision of the level of normative values of environmental impact of the corresponding negative factors by actively suppressing them (for example, the installation of catalysts and neutralizers in the exhaust system of a vehicle; installation of a device for cleaning dust from the air removed from the cement clinker crushing plant, etc.).

In further studies based on the study of factors negatively affecting the environment from the standpoint of the theory of dispersed systems $[9,10,11]$, we suggest to develop a physical and energy scientific concept for the study of remediation measures and 
corresponding environmental technologies. This approach should be based on considering the parameters characterizing properties and energy state of each factor affecting the environment, grouping these parameters, building process models of environmental pollution by the influencing factor and suppressing this factor, followed by the choice of appropriate remediation measures $[12,13]$.

In further research, from the entire complex of measures, we examined precisely the group of special remediation measures (Figure 2), which are based on environmental technologies. It should be noted that the concept of "event" is complex and has a broader meaning in comparison with the concept of "technology", which has Greek roots, the meaning most closely corresponds to the concept of "skill" and characterizes the sequence of actions, covering their extremely wide profile and spectrum, emphasizing the exceptional variety of technological methods, techniques, tools, approaches in the field of engineering, chemistry, biology, agriculture, medicine and other natural sciences. The same activity can be implemented with different technologies (with varying degrees of skill). One of the basic principles, provided that environmental technologies are used, should be considered to ensure their maximum safety for all living organisms and, accordingly, their minimum negative impact on the environment. Unfortunately, these principles are not always implemented.

In terms of the scale of the zone of their effective action, environmental technologies can be divided into three groups: local, regional and global, since in modern conditions the scaling of environmental technologies is extremely important $[14,15]$.

\section{Conclusion}

Noting the function and role of environmental technologies in the group of remediation measures, attention should be paid to their difference from any others in that their main task is to restore and monitor the native (natural initial) state of the environment with the inherent biodiversity characteristic of any region of the planet. The variety of remediation measures is explained by the corresponding variety of factors polluting the environment, which were previously also systematized by us according to various classification criteria: by origin, by state of aggregation, by toxicity, by purpose and field of application.

That is why our proposed principles for improving the global monitoring system, as well as a scientifically grounded classification of environmental measures aimed at suppressing factors affecting the environment are scientific prerequisites for developing an integrated approach to the selection of remediation measures and environmental technologies for their implementation.

\section{References}

1. S.S. Lidzhieva, Ya.A. Akhrestina, V.V. Erdneeva, The current state of the problem of environmental pollution in the role of humanitarian and environmental education at the present stage of development of society (2019)

2. V.I. Bespalov, O.N. Paramonova, German/English/Russian language, peerreviewed journal and is published monthly 1(1/1), 202-204 (2013)

3. O. Paramonova, V. Bespalov, O. Gurova, N. Krivtsova, MATEC Web Conf. 170 (2018) DOI: https://doi.org/10.1051/matecconf/201817004014

4. V.I. Bespalov, O.S. Gurova, O.N. Paramonova, Security technogenic and natural systems 2, 43-52 (2020) https://doi.org/10.23947/2541-9129-2020-2-43-52

5. A. Palliyarayil et al., Emergent Materials, 1-37 (2021) 
6. M. Qin et al., Nature Sustainability 4(2), 129-137 (2021)

7. M. Qin, B.N. Murphy, K.K. Isaacs et al., Nature Sustainability 4(2), 129-137 (2021)

8. N. Gaur, K. Narasimhulu, Y. Pydi Setty, Journal of cleaner production 198, 1602-1631 (2018)

9. K. Yan et al., Advanced Functional Materials 31(12), 2008227 (2021)

10. N. Samarskaya, O Paramonova, E Lysova, International science and technology conference "Earth science" IOP Conf. Series: Earth and Environmental Science 666, 042072 (2021) https://iopscience.iop.org/article/10.1088/1755-1315/666/4/042072/pdf

11. N. Samarskaya, E. Lysova, O. Paramonova, N. Yudina, IOP Conference Series: Earth and Environmental Science 459(2), $022074 \quad$ (2020) https://iopscience.iop.org/article/10.1088/1755-1315/459/2/022074

12. N.S. Samarskaya, O.N. Paramonova, N.V. Yudina, 2nd International Conference on Industrial Engineering (ICIE-2016) 150 (2016) http://www.sciencedirect.com/science/ article/pii/S1877705816316034

13. N.S. Samarskaya, N.V. Yudina, A.V. Koroleva, Scientific almanac of the Black Sea Countries 10(2) (2017) http://science-almanac.ru/documents/151/2017-02-13-UdinaLysova-Koroleva.pdf

14. N. Samarskaya, O. Paramonova, E. Lysova, IOP Conference Series: Earth and Environmental Science, International science and technology conference "Earth science" 666 (2020) doi:10.1088/1755-1315/666/4/042072

15. N.V. Yudina, L.A. Dolzhenko, International Conference on Construction and Architecture: Theory and Practice of Industry Development (2018) https://www.scientific.net/conference-2676 